\title{
On reduced pairs of bounded closed convex sets
}

\author{
Jerzy GRZYBOWSKI and Ryszard URBAŃSKI \\ Faculty of Mathematics and Computer Science \\ Adam Mickiewicz University \\ Umultowska 87, PL-61614 Poznań, Poland \\ jgrz@amu.edu.pl \\ rich@amu.edu.pl
}

Recibido: 12 de Septiembre de 2003

Aceptado: 23 de Febrero de 2002

\section{ABSTRACT}

In this paper certain criteria for reduced pairs of bounded closed convex set are presented. Some examples of reduced and not reduced pairs are enclosed.

2000 Mathematics Subject Classification: 52A07, 26 A27.

Key words: Convex analysis, pairs of convex sets

Let $X=(X, \tau)$ be a topological vector space over the field $\mathbb{R}$. Let $\mathcal{K}(X)[\mathcal{B}(X)]$ be a family of all nonempty compact [bounded closed ] convex subsets of $X$. For any $A, B \subset X$ the Minkowski sum is defined by $A+B=\{a+b \mid a \in A$ and $b \in B\}$. Since $A+B$ is not always closed [4], [9] we define $A+B=\overline{A+B}$ for $A, B \in \mathcal{B}(X)$. It was showed in [9] that for $A, B, C \in \mathcal{B}(X)$ the inclusion $A+B \subset B+C$ implies $A \subset C$. From this it follows that $\mathcal{B}(X)$ together with " + " is a semigroup satisfying the law of cancellation, i.e. $A+B=B+C$ implies $A=C$.

For $(A, B),(C, D) \in \mathcal{B}^{2}(X)$, let $(A, B) \sim(C, D)$ if and only if $A \subset C, B \subset D$ and $(A, B) \sim(C, D)$. The relation " $\sim$ " is an equivalence relation in $\mathcal{B}^{2}(X)$ and " $\leq$ " is an ordering in the equivalence class $[A, B]$ of any pair $(A, B)$. It should be mentioned that the space $\mathcal{K}(X) / \sim, \mathcal{K}(X)=\{A \in \mathcal{B}(X) \mid A$ is compact $\}$, plays important role in quasidifferential calculus [2].

The set $A \in \mathcal{B}(X)$ is called a polytope if $A$ is convex hull of a finite set. If $A, B \in \mathcal{B}(X)$ then $A \vee B$ is the convex hull of $A \cup B$.

It was proved in [6] that if $A, B \in \mathcal{K}(X)$, then there exists minimal element $(C, D)$ in $[A, B]$ such that $(C, D) \leq(A, B)$. From [3], [8] we know that if $(A, B),(C, D) \in$ $\mathcal{K}^{2}(X)$, are two minimal pairs in $[A, B]$ and $\operatorname{dim} X \leq 2$ then $C+x, D=B+x$. 
Let $(A, B) \in \mathcal{B}^{2}(X)$. The pair $(A, B)$ is called reduced if for any $(C, D) \in[A, B]$ there exists $M \in \mathcal{B}(X)$ such that $C=A+M$ and $D=B+M$. Let us notice that every reduced pair is minimal. Every minimal pair is reduced in $X=\mathbb{R}$ (see, [6]).

Let $A \in \mathcal{K}(X), f \in X^{*}$. Then $H_{f} A=\left\{x \in A \mid f(x)=\max _{y \in A} f(y)\right\}$.

The set $A \in \mathcal{B}(X)$ is called a summand of $B \in \mathcal{B}(X)$ if there exists $M \in \mathcal{B}(X)$ such that $B=A+M$.

W. Weil has proved in [11] the following lemma.

Lemma. Let $A, B \in \mathcal{K}\left(\mathbb{R}^{n}\right)$ and $A$ be a convex polytope. Then $A$ is a summand of $B$ if an only if each one-dimensional face $H_{f} A$ is contained in a translate of the corresponding face $H_{f} B$.

Theorem 1. Let $A, B \in \mathcal{K}\left(\mathbb{R}^{n}\right)$ and $A$ be a convex polytope such that card $H_{f} B=1$ for each one-dimensional face $H_{f} A$. Then the pair $(A, B)$ is reduced.

Proof. Let $\left(C, D \in[A, B]\right.$. Then $A+D=B+C$. Let $f \in\left(\mathbb{R}^{n}\right)^{*}$ and $H_{f} A$ be onedimensional face of $A$. Then, by virtue of the formula of the addition of faces, we have

$$
H_{f} A+H_{f} D=H_{f} B+H_{f} C .
$$

According to the assumption, $H_{f} B=\{b\}$ for some $b \in \mathbb{R}^{n}$. Then $H_{f} A \subset b-d+H_{f} C$, where $d \in H_{f} D$. Applying Lemma, we obtain that $C=A+M$ for some $M \in \mathcal{K}\left(\mathbb{R}^{n}\right)$. Hence, from the law of cancellation, it follows that $D=B+M$.

Theorem 2. Let $A, B \in \mathcal{K}\left(\mathbb{R}^{2}\right)$ be a reduced pair. Then $\operatorname{card} H_{f} B=1$ for each one-dimensional face $H_{f} A$.

Proof. Let us assume that $\operatorname{dim} H_{f} B=\operatorname{dim} H_{f} A=1$ for some $f \in\left(\mathbb{R}^{2}\right)^{*}$. Then there exists an interval $I$ and a triangle $T$ such that length of $I$ is not greater than both lengths of $H_{f} A$ and $H_{f} B$, and $H_{-f} T=I$. If $H_{f} T=\{b\}$ then $H_{f}(A+T)=$ $H_{f} A+b, H_{-f}(A+T)=H_{-f} A+I, H_{f}(B+T)=H_{f} B+b$ and $H_{-f}(B+T)=H_{-f} B+I$. Hence $I$ is a summand of both $A+T$ and $B+T$, and $A+T=A^{\prime}+I, B+T=B^{\prime}+I$ for some $A^{\prime}, B^{\prime} \in \mathcal{K}\left(\mathbb{R}^{2}\right)$. Then $\left.A^{\prime}, B^{\prime}\right) \in[A, B]$, and since $H_{f} A$ is not a summand of $H_{f} A^{\prime}$ then $A$ is not a summand of $A^{\prime}$. Therefore, $(A, B)$ is not reduced.

Proposition 1. Let $(A, B),(C, D),(E, F) \in \mathcal{B}^{2}(X)$ and $A=C+E, B=D+F$. If the pair $(A, B)$ is reduced then both $(C, D)$ and $(E, F)$ are reduced.

Proof. Let $\left(C^{\prime}, D^{\prime}\right) \in[C, D]$. Then $C^{\prime} \dot{+} D=C \dot{+} D^{\prime}$, and we have

$$
A \dot{+} D \dot{+} F \dot{+} D^{\prime}=A \dot{+} B \dot{+} D^{\prime}=C \dot{+} E \dot{+} B \dot{+} D^{\prime}=E \dot{+} B \dot{+} C^{\prime} \dot{+} D .
$$


Hence $A+F \dot{+} D^{\prime}=B \dot{+} E \dot{+} C^{\prime}$. From the assumption, it follows that $E \dot{+} C^{\prime}=$ $A+M$ and $F+D^{\prime}=B+M$ for some $M \in \mathcal{B}(X)$. Then $E+C^{\prime}=C+E+M$ and $F \dot{+} D^{\prime}=D+F+M$. Hence $C^{\prime}=C \dot{+} M$ and $D^{\prime}=D+M$.

Proposition 2. Let $A, B \in \mathcal{B}(X)$. If the pair $(A \vee B, A+B)$ is reduced then $(A \vee B, B)$ is also reduced.

Proof. Since $(A \vee B, A+B)=(A \vee B, B)+(\{0\}, A)$ then applying Proposition 1 we obtain our Proposition.

Let $A, B \in \mathcal{B}(X)$. We call the pair $(A, B)$ convex if $A \cup B$ is convex. We call $(A, B)$ convexly reduced if for any convex pair $(C, D)$ in $[A, B]$ there exists $M \in \mathcal{B}(X)$ such that $C=A+M$ and $D=B+M$.

Theorem 3. The convex pair $(A, B) \in \mathcal{B}^{2}(X)$ is convexly reduced if and only if $(A \cap B, A \cup B)$ is reduced.

Proof. $\Rightarrow$ ) Let the pair $(A, B)$ be convexly reduced and $(F, G) \in[A \cap B, A \cup B]$. From [4],[10] it follows that there exists $\left(A_{0}, B_{0}\right) \in[A, B]$ such that $A_{0} \cap B_{0}=F$ and $A_{0} \cup B_{0}=G$. From the assumption, $A_{0}=A+M$ and $B_{0}=B+M$ for some $M \in \mathcal{B}(X)$. Then $F=A_{0} \cap B_{0}=A \cap B+M$ and $G=A_{0} \cup B_{0}=A \cup B \dot{+} M$. Therefore, the pair $(A \cap B, A \cup B)$ is reduced.

$\Leftrightarrow)$ Let $(A \cap B, A \cup B)$ be reduced, $(C, D) \in[A, B]$ and $C \cup D$ be convex. Then $A \dot{+}$ $D=B \dot{+} C=A \cap B \dot{+} C \cup D=C \cap D \dot{+} A \cup B$, [see [10]]. Hence $C \cap D=A \cap B \dot{+} M$ and $C \cup D=A \cup B+M$ for some $M \in \mathcal{B}(X)$. From the law of cancellation, we obtain $C=A+M$ and $D=B+M$.
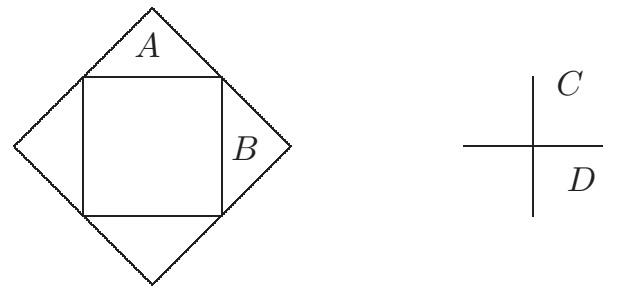

The pair $(A, B)$ is convexly reduced and $(A, B) \sim(C, D)$. 
Theorem 4. Let $A, B \in \mathcal{B}(X)$. If $(A \vee B, B)$ is a reduced pair then the pair $(A, B)$ is reduced.

Proof. Let $(C, D) \in[A, B]$. Then $A \dot{+} D=B \dot{+} C$. Therefore,

$$
D \dot{+} A \vee B=(A \dot{+} D) \vee(B \dot{+} D)=(B \dot{+} C) \vee(B \dot{+} D)=B \dot{+} C \vee D
$$

Since the pair $(A \vee B, B)$ is reduced then $D=B+M$ for some $M \in \mathcal{B}(X)$. From the law of cancellation ([9]) $C=A+M$.

The pair $(A, B)$ is convexly reduced and $(A, B) \sim(C, D)$. The pair $(A, B)$ is also reduced and the class $[A, B]$ is convex, that is $C \cup D$ is convex for any $(C, D) \in[A, B]$ ([4]).

In [5] the following theorem was proved:

Theorem 5. Let $A, B \in \mathcal{K}\left(\mathbb{R}^{n}\right)$ and $A$ be a polytope with nonempty interior. Let card $H_{f} B=1$ for each face $H_{f} A$ such that $\operatorname{dim} H_{f} A=n-1$. Then the pair $(A, B)$ is minimal.

For $n=2$, Theorem 1 and Theorem 5 have equivalent assumptions, hence Theorem 1 is stronger than Theorem 5 . For $n=3$, the assumption of Theorem 5 is weaker than the assumption of Theorem 1 . The following example shows that generally we cannot replace the assumption in Theorem 1 with the assumption from Theorem 5 .

Example. Let $A=[-1,1]^{3}$ and $B=A \vee(0,0,3 / 2) \vee(0,0,-3 / 2) \vee(0,3 / 2,0) \vee(0,-3 / 2,0) \vee(3 / 2,0,0) \vee(-3 / 2,0,0)$. Let us notice that if $\operatorname{dim} H_{f} A=2$ then $\operatorname{card} H_{f} B=1$. Let $I=(1,0,0) \vee(0,1,0)$. Let $A^{\prime}=$ $(A+I) \vee(5 / 3,5 / 3,0)$ and $B^{\prime}=(B+I) \vee(5 / 3,5 / 3,0)$. We have $\left(A^{\prime}, B^{\prime}\right) \sim(A+I, B+$ $I) \sim(A, B)$. Let us notice that $H_{f} A^{\prime}=(5 / 3,5 / 3,0)$ and $H_{f} A=(1,1,-1) \vee(1,1,1)$ for $f(x, y, z)=x+y$. Then $A$ is not a summand of $A^{\prime}$. The pair $(A, B)$ is not reduced. 

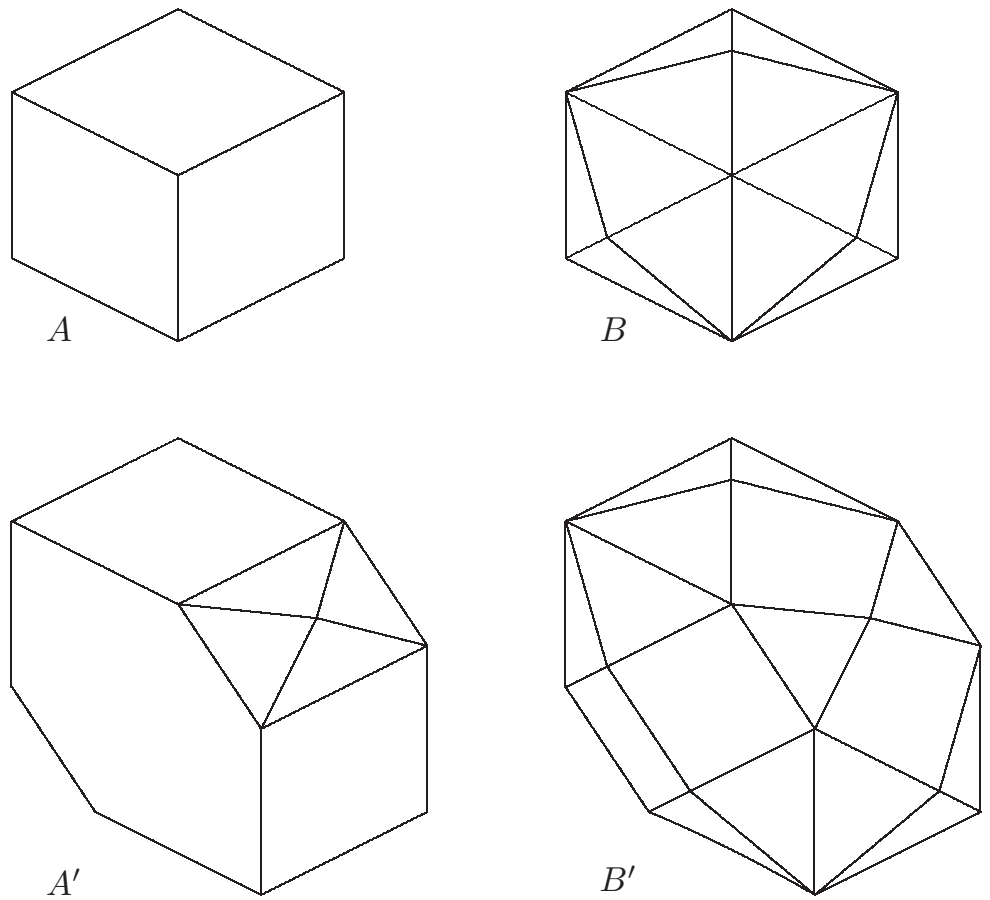

\section{References}

[1] C. Bauer, Minimal and reduced pairs of convex bodies, Geom. Dedicata 62 (1996), 179192.

[2] V. F. Demyanov and A. M. Rubiov, Quasidifferential Calculus, Optimization Software Inc., Springer-Verlag, New York, 1986.

[3] J. Grzybowski, Minimal pairs of compact sets, Arch. Math. 63 (1994), 173-181.

[4] J. Grzybowski and R. Urbański, Minimal pairs of bounded closed convex sets, Studia Math. 126 (1997), 95-99.

[5] J. Grzybowski, R. Urbański and M. Wiernowolski, On Common Summands and Antisummands of Compact Convex Sets, Bull. Polish. Acad. Sci. Math. 47 (1999), 69-76.

[6] D. Pallaschke, S. Scholtes and R. Urbański, On minimal pairs of convex compact sets, Bull. Polish Acad. Sci. Math. 39 (1991), 1-5.

[7] R. Schneider, On asymmetry classes of convex bodies, Mathematika 21 (1974), 12-18.

[8] S. Scholtes, Minimal pairs of convex bodies in two dimensions, Mathematika 39 (1992), $267-273$.

[9] R. Urbański, A generalization of the Minkowski-Rådström-Hörmander Theorem, Bull. Acad. Polon. Sci. Sér. Sci. Math. Astronom. Phys. 24 (1976), 709-715. 
[10] R. Urbański, On minimal convex pairs of convex compact sets, Archiv der Mathematic 67 (1996), 226-238.

[11] W. Weil, Decomposition of convex bodies, Mathematika 21 (1974), 19-25. 